\title{
EQUIVARIANT HOMOTOPY THEORY AND MILNOR'S THEOREM
}

\author{
BY
}

\author{
STEFAN WANER
}

\begin{abstract}
The foundations of equivariant homotopy and cellular theory are examined; an equivariant Whitehead theorem is proved, and the classical results by Milnor about spaces with the homotopy-type of a CW complex are generalized to the equivariant case. The ambient group $G$ is assumed compact Lie. Further results include equivariant cellular approximation and the procedure for replacement of an arbitrary $G$-space by a $G-C W$ complex.
\end{abstract}

This is the first of a series of three papers based on the author's thesis [Wa1], the object of which is to discuss equivariant homotopy theory in general, and equivariant fibrations in particular, culminating in classification theorems for the various categories of equivariant fibrations and bundles.

In the present paper, we discuss the foundations of equivariant homotopy theory and cellular theory and prove an equivariant version of Milnor's theorem on spaces having the homotopy type of CW complexes, where we allow a compact Lie group $G$ to act everywhere. The second paper in this series, Equivariant fibratons and transfer sets up the background for the study of $G$-fiber spaces and equivariant stable homotopy theory and contains a description of the equivariant transfer for equivariant fibrations with compact fiber. In the third paper, Equivariant classifying spaces and fibrations, the geometric bar construction is used to construct explicit classifying spaces for equivariant bundles and fibrations, these results depending heavily on the equivariant cellular theory presented here. Also in preparation is a fourth paper which will sequel the present series and will deal with the classificaton of oriented $G$-spherical fibrations and bundles [Wa2].

The three papers are divided as follows:

Equivariant homotopy theory and Milnor's Theorem

1. Notations and definitions

2. Equivariant homotopy groups

3. Equivariant cellular theory

4. Milnor's Theorem

5. Approximation of $G-C W$ complexes by $G$-simplicial complexes

6. Finite dimensional $G$-simplicial complexes are $G$-equilocally convex

7. Reasonable $G$ ELC spaces are dominated by $G-C W$ complexes

Received by the editors September 7, 1978 and, in revised form, January 15, 1979.

AMS (MOS) subject classifications (1970). Primary 54H15.

Key words and phrases. $G$-homotopy, fixed point set, isotopy subgroup, G-CW complex, G-equilocally convex, invariance of colimits. 
Equivariant fibrations and transfer

1. Equivariant fibrations and quasifibrations

2. Equivariant quasifibrations and gammafication

3. Equivariant Spanier-Whitehead duality and stable homotopy

4. Equivariant fiberwise duality

5. Equivariant transfer

6. Equivariant cellular theory and fibrations

\section{Equivariant classifying spaces and fibrations}

1. Equivariant fibrations and categories of fibers

2. Construction of equivariant classifying spaces

3. Based fibrations, bundles and stable fibrations

I am extremely grateful to Professor Peter May, my thesis supervisor, for his assistence and inexhaustible patience, and to Professors Mel Rothenberg and Dick Lashof for their invaluable suggestions and encouragement.

0. Introduction. Equivariant homotopy theory has traditionally been handled in two distinct ways:

(1) "Strict" $G$-homotopy theory, in which all homotopies are through $G$-maps, and hence inverses are also $G$-maps;

(2) "sh" $G$-theory, in which one deals with $G$-maps which are homotopy equivalences, although the inverses need not be $G$-maps, as in, for example, [LW].

The latter suggest the unlikelihood of a Whitehead theorem for $G$-spaces, and hence the unlikelihood of a useful notion of a $G-C W$ complex unless $G$ is discrete. Furthermore, the former approach seems to be a more natural generalization of nonequivariant homotopy theory.

The purpose of this paper will be to establish the foundations of "strict" $G$-homotopy theory for a compact Lie group $G$. In order to do this, various gaps in the literature must be filled, especially in the area of cellular and simplicial theory. The major concern of this paper will be to prove an equivariant version of Milnor's theorem on spaces with the homotopy type of a CW complex [Mi1]. When $G$ is finite, this is fairly straightforward, though it will be seen that the general case is fairly intricate.

As far as the other results of this paper are concerned, it will be seen that the generalized treatment of homotopy theory in [Mal] readily adapts to the equivariant case. In particular, we obtain an equivariant version of Whitehead's theorem (our proof being simpler than that of Matumoto [Mo1]).

The concept of a $G-\mathrm{CW}$ complex is due to Matumoto [Mo1] (and to Bredon in the case of finite $G$ ).

We shall be working in the realm of unbased $G$-spaces, though there will be indication as to how to adapt the theory to the realm of based $G$-spaces where necessary. 
1. Notations, definitions and categorical matter. $\mathscr{U}$ will denote the category whose objects are compactly generated weak Hausdorff spaces and whose morphisms are continuous functions. If $G$ is a topological group, $G \mathscr{Q}$ will denote the category whose objects are spaces in $\mathscr{U}$ with specified right $G$-actions, and whose morphisms are those morphisms in $\mathscr{U}$ which commute with the specified $G$-actions.

All coset spaces will be understood to be right coset spaces.

Observe that the categories $\mathcal{U}$ and $G \mathscr{U}$ have products, pushouts, pullbacks and all the categorical limits and colimits. In addition, each contains the unit interval $I$ (which, on $G \mathscr{U}$, has the trivial $G$ action) and thus a notion of homotopy (called $G$-homotopy in $G \mathscr{U}$ ) and of associated homotopy category which will be denoted by $h \mathscr{Q}$ and $h G \mathscr{Q}$ respectively. Thus a $G$-homotopy equivalence is a $G$-map $f: X \rightarrow Y$ such that there exists a $G$-map $g: Y \rightarrow X$ with $f g$ and $g f$ each homotopic to the identity through $G$-maps.

The categories of $n$-tuples in $\mathscr{U}$ and $G \mathscr{U}$ will be denoted by $\mathcal{U}(n)$ and $G \mathscr{U}(n)$.

थ will denote the full subcategory of $\mathcal{U}$ whose objects have the homotopy type of a CW complex. Similarly, $G \mho$ will denote the full subcategory of $G \mathscr{U}$ whose objects have the $G$-homotopy type of $G-\mathrm{CW}$ complexes (defined in §3). This leads again to categories $h W$ and $h G \mho$.

If $H$ is a closed subgroup of $G$, we have forgetful functors $\mathscr{F}_{H}: G \mathscr{Q} \rightarrow H \mathscr{Q}$, and

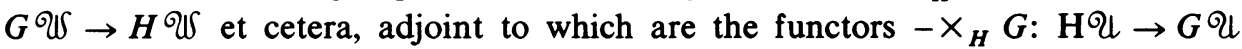
which associate to a space $X$ the twisted products $X \times{ }_{H} G$. Thus: $H$ थ $\left(X, \mathscr{F}_{H} Y\right)$ $\cong G \mathscr{U}\left(X \times{ }_{H} G, Y\right)$. Furthermore, the functors $(-)^{H}: G \mathscr{Q} \rightarrow \mathcal{Q}$ and $-X G / H$ : $\mathscr{Q} \rightarrow G \mathscr{Q}$ also form an adjoint pair, so that $\mathcal{Q}\left(X, Y^{H}\right) \simeq G \mathcal{Q}(X \times G / H, Y)$, where $X^{H}$ denotes the set of points in $X$ fixed by $H$ topologized as a subspace of $X$.

When there is no danger of ambiguity, $h G \mathcal{Q}(X, Y)$ will be denoted by $[X, Y]$, and sometimes by $[X, Y]_{G}$.

$I^{n}$ will denote the unit $n$-cube, and $\dot{I}^{n}$ its boundary. The basic facts about cofibrations apply readily to $G$-cofibrations, namely maps which satisfy the $G$-homotopy extension property.

The following results from [Ma1] apply formally to the category $G \mathscr{U}$.

TheOREM 1.1 (INVARIANCE OF PUSHOUTS). Suppose that we have a commutative diagram:

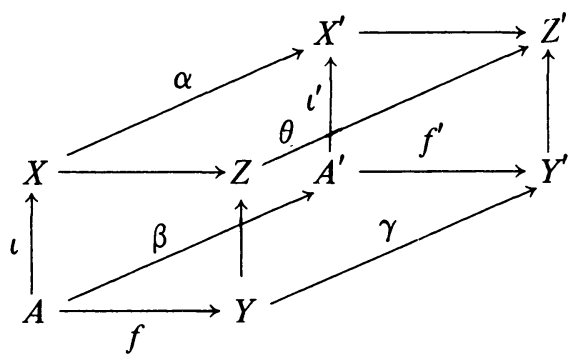

in which $\iota$ and $\iota^{\prime}$ are $G$-cofibrations, $f$ and $f^{\prime}$ are arbitrary $G$-maps, and $\alpha, \beta$ and $\gamma$ are equivariant homotopy equivalences. Then $\theta$ is also a G-homotopy equivalence ( $\theta$ being the induced map on pushouts). 
TheOREM 1.2 (INVARIANCE OF COLIMITS OVER COFIBRATIONS). Suppose given a homotopy commutative diagram

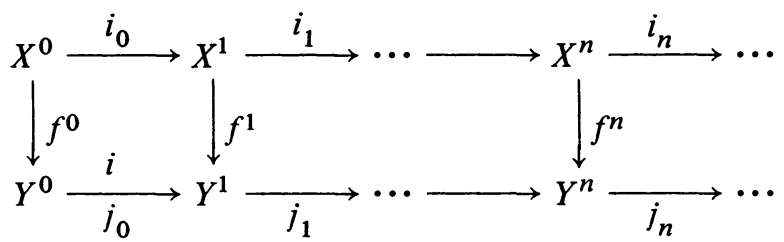

in $G$, where the $i_{m}$ and $j_{m}$ are $G$-cofibrations and the $f^{m}$ are $G$-homotopy equivalences. Then the map colim $f^{n}$ : colim $X^{n} \rightarrow \operatorname{colim} Y^{n}$ is a G-homotopy equivalence.

\section{Equivariant homotopy groups.}

Definition 2.1. Let $(X, Y)$ be in $G थ(2)$, and let $\phi: G / H \rightarrow Y$ be a $G$-map with $H$ an arbitrary closed subgroup of $G$. For each $n>1$, define

$$
\pi_{n}^{H}(X, Y, \phi)=h G \mathcal{Q}\left(\left(I^{n}, I^{n},\{0\}\right) \times G / H,(X, Y, Q)\right)
$$

where $Q$ is the image of $\phi$, and all the homotopies are relative to $\phi: G / H \rightarrow Q .\{0\}$ specifies the basepoint in $I^{n}$. Also define $\pi_{n}^{H}(X, \phi)=\pi_{n}^{H}(X, Q, \phi)$ with the understanding that the homotopies here are taken relative to $\phi \circ \pi_{2}: I^{n} \times G / H \rightarrow Q$, where $\pi_{2}$ denotes the second projection.

For $n=0$, we adopt the convention $I^{0}=\{0,1\}$ and $i^{0}=\{0\}$ and proceed as above.

The operations $\pi_{n}^{H}$ extend to functors on the appropriate categories (modulo adjustment at $\phi$ ). We shall recklessly omit $\phi$ from the notations unless it becomes necessary to consier the base orbit map. When considering varying ambient groups $G$, we add $G$ to the notation, writing $\pi_{n}^{H}(X, Y, \phi ; G)$.

The adjunctions mentioned in $\$ 1$ allow us to reduce many problems of equivariant homotopy theory to their nonequivariant analogues, as is demonstrated by the following proposition.

Proposition 2.2. There are natural equivalences $\pi_{n}^{H}(X) \simeq \pi_{n}\left(X^{H}\right)$ and $\pi_{n}^{H}(X, Y)$ $\simeq \pi_{n}\left(X^{H}, Y^{H}\right)$ which preserve the (evident) group structures when $n>1$ (for the absolute case; the relative case requires $n \geq 2$ ).

Proof is omitted, since the proposition is an immediate consequence of the adjunction relations mentioned above.

As a direct consequence of either 2.2 or the definitions, we obtain the expected long exact sequences:

Proposition 2.3. For $(X, Y)$ and $H$ as in 2.1 , there exist natural boundary maps $\partial$ and long exact sequences

$$
\cdots \rightarrow \pi_{n}^{H}(X, Y) \stackrel{\partial}{\rightarrow} \pi_{n-1}^{H}(Y) \rightarrow \pi_{n-1}^{H}(X) \rightarrow \cdots \rightarrow \pi_{0}^{H}(Y) \rightarrow \pi_{0}^{H}(X)
$$

of groups up to $\pi_{1}^{H}(Y)$ and of pointed sets thereafter. 
Definition 2.4. A map $e:(X, Y) \rightarrow\left(X^{\prime}, Y^{\prime}\right)$ of $G$-pairs is a $G$-n-equivalence if $e^{H}:\left(X^{H}, Y^{H}\right) \rightarrow\left(X^{\prime H}, Y^{\prime H}\right)$ is an $n$-equivalence for each closed subgroup $H$ of $G$; a map $e$ will be called a $G$-weak equivalence if it is a $G$ - $n$-equivalence for each $n>0$.

Similarly, a map $e: X \rightarrow Y$ in $G \mathscr{Q}$ is a $G(n)$-equivalence if each $e^{H}$ is an n-equivalence.

Proposition 2.5. The functors $\mathscr{F}_{H}: G \mathscr{U} \rightarrow H \cup$ preserve weak n-equivalence for all $n$.

Proof. This is an immediate consequence of 2.2 .

3. Equivariant cellular theory. Some of this material is presented in [Mo1], where a version of the $G$-Whitehead theorem is proved. It is shown here how this theorem, and more, follows from the general treatment of classical homotopy theory in [Ma1].

Let $G$ be a topological group (not necessarily compact).

Definition 3.1. A $G$-CW complex is a space $X$ in $G \mathscr{U}$ with a decomposition $X=\operatorname{colim} X^{n}$, where

$$
X^{0}=\amalg_{\alpha \in A_{0}} G / H_{\alpha}, \quad X^{n+1}=X^{n} \cup_{\phi}\left(\underset{\alpha \in A_{n}}{\amalg} D^{n} \times G / H_{\alpha}\right)
$$

for some $G$-map $\phi: \amalg_{\alpha \in A_{n}} S^{n} \times G / H_{\alpha} \rightarrow X^{n}$, and $\left\{H_{\alpha}\right\}_{\alpha \in A_{n}}$ is a collection of closed subgroups of $G$.

Recall that a $G$-orbit $Q_{0}$ is said to be of type $<$ that of a $G$-orbit $Q_{1}$ if there is a $G$-map $Q_{1} \rightarrow Q_{0}$. (We sometimes say $T\left(Q_{0}\right)<T\left(Q_{1}\right)$.) This is equivalent to the assertion that the isotropy subgroup of every point in $Q_{1}$ is contained in some conjugate of the isotropy subgroup of some point in $Q_{0}$. For example, $T(G / H)<$ $T(G / K)$ if and only if $H \subset K^{a}$ for some $a \in G$. (Here $K^{a}$ denotes $a^{-1} K a$, for all coset spaces are right coset spaces.) (See [Br1] for details.)

A $G$-subcomplex and a relative G-CW complex are now defined in the obvious way. Observe that the orbits in $X^{n+1}$ must be $>$ those to which they attach in $X^{n}$.

Examples of $G-C W$ complexes abound, and include all smooth $G$-manifolds for $G$ a compact Lie group (see [Mo1] and [Mo2] for details, our $G-\mathrm{CW}$ complexes being equivalent to his).

The following technical lemma is due to May [Ma2, 3.5.1] and is adapted here to the equivariant case.

LEMMA 3.2 (MAY). Suppose that $e: Y \rightarrow Z$ is a $G$-n-equivalence. Then we can complete the following diagram in $G \mathscr{U}$ :

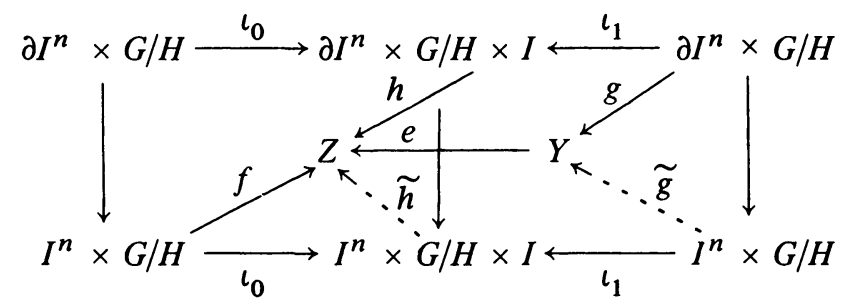


Proof. The maps $f$ and $h$ fit together to yield an element of $\pi_{n}^{H}(M e, Y)=0$. The resulting null homotopy easily gives $\tilde{g}$ and $\tilde{h}$.

TheOREM 3.3 ( $G$-HELP). If $(X, A)$ is a relative $G$-CW complex of dimension $<n$ and $e: Y \rightarrow Z$ is a $G$-n-equivalence; then we can complete the following diagram in $G$ U:

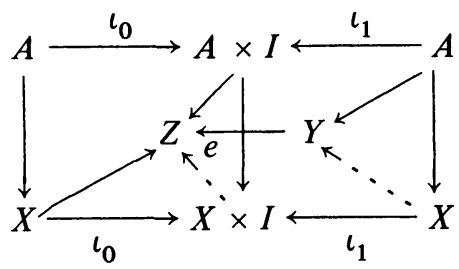

Proof. This follows by induction on $\operatorname{dim}(X, A)$, applying 3.2 cell-by-cell at each stage.

Due to the fact that we have insisted that the diagram above commute on the nose, the $n=\infty$ variant of Theorem 3.3 follows formally.

We now prove the Whitehead Theorem.

Theorem 3.4 (G-WhiteheAD). (i) Suppose that $X$ is a G-CW complex, and that $e$ : $Y \rightarrow Z$ is a $G$-n-equivalence. Then $e_{*}:[X, y] \rightarrow[X, Z]$ is an isomorphism if $\operatorname{dim} X$ $<n$ and an epimorphism if $\operatorname{dim} X=n$.

(ii) If $e: Y \rightarrow Z$ is a weak $G$-equivalence, and if $X$ is any (possibly infinite) $G-C W$ complex, then $e_{*}[X, Y] \rightarrow[X, Z]$ is an isomorphism.

Proof. (i) Apply $G$-HELP to the pair $(X, \phi)$ for surjectivity, and to the pair $(X \times I, X \times \dot{I})$ for injectivity.

(ii) This is identical to (i), in view of the remark after 3.2 .

Theorem 3.5 (G-Cellular APProximation). Suppose that $X$ is a G-CW complex, and that $A$ is a sub-G-CW complex of $X$. Then, if $f: X \rightarrow Y$ is a $G$ map which is cellular when restricted to $A$, we can $G$-homotope $f, \operatorname{rel} f \mid A$ to a $G$-cellular map $g$ : $X \rightarrow Y$.

Proof. We first observe that the inclusions $\iota_{n}: Y^{n} \rightarrow Y$ are $G$ - $n$-equivalences by essentially the same argument as in the $G$-trivial case. The result then follows by an easy adaptation of the proof given in [Ma1, 1.5.4].

We recall the following terminology from [Ma1, 2.1]:

Definition 3.6. Let $\mathcal{Q}$ be a category. Then a set $\mathcal{G}$ of objects of $\mathcal{Q}$ is said to be compact if, for each sequence of maps $X_{n} \rightarrow X_{n+1}$, there exists a weak colimit Tel $X_{n}$ such that the natural map colim $\mathbb{Q}\left(J, X_{n}\right) \rightarrow \mathbb{Q}\left(J, \mathrm{Tel} X_{n}\right)$ is a bijection for every $J$ in $\mathcal{G}$.

Whether or not $G$ is compact, the set $\mathcal{G}=\left\{G / H \times S^{n}, n>0\right.$ and $H$ a closed subgroup of $G\}$ is a compact set in $h G \mathscr{U}$. In view of this, we obtain the following variant of [Ma1, 3.8.1] (cf. [II1] for finite $G$ ). 
Theorem 3.7. For any space $X$ in $G \mathcal{Q}$, there is a G-CW complex $\Gamma X$ and a weak G-equivalence $\gamma: \Gamma X \rightarrow X$. If $f: X \rightarrow Y$ is a map, and if $\gamma: \Gamma Y \rightarrow Y$ is such a G-CW approximation, then there is a map $\Gamma f: \Gamma X \rightarrow \Gamma Y$, unique up to G-homotopy, such that $\gamma \circ \Gamma f=f \circ \gamma$.

Proof. One has to verify that the Brown construction [Ma1, §2] yields a $G-C W$ complex $\Gamma X$. But the nonequivariant cellular decompositions of weak coequalizers easily go over to the $G$ case. Further, the universal map $\gamma: \Gamma X \rightarrow X$ is a weak $G$-equivalence by construction of $\Gamma X$.

(We have avoided the use of the geometric realization of the singular chain complex as this is clearly inappropriate for nondiscrete groups $G$; that is, there is no $G$-action on this realization. Perhaps an alternative treatment could involve Illman's $G$-simplices; maps $G / H \times \Delta^{n} \rightarrow X$ glued together according to his identifications. A technical problem might arise here, as we would lose comparison between the cardinality of the resulting complex, which would be vast, and that of the $G$-homotopy groups of $X$ over which we have control when $X$ is reasonable.)

We now include a statement to the effect that the forgetful functors $\mathscr{F}_{H}$ preserve $G$-CW structure up to homotopy. Assume that $G$ is a compact Lie group.

Proposition 3.8. Let $\mathscr{F}_{H}: G \mathscr{U} \rightarrow H \mathcal{U}$ denote the forgetful functor. Then $\mathscr{F}_{H}$ restricts to a functor $\tilde{\mathscr{F}}_{H}: G$ W $\rightarrow H$ W.

Proof. Assume given a $G$-CW complex $X . X^{0}$ has the $H$-homotopy type of an $H$-CW complex by Milnor's Theorem in $\S 4$, since $G$-orbits are $H$-equilocally convex for any closed subgroup $H<G$. (See §4.) Thus we assume inductively that we are given $H$-CW complexes $\bar{X}^{m}$ for $m \leqslant n, G$-cellular inclusions $i^{m}: \bar{X}^{m-1} \rightarrow$ $\bar{X}^{m}$, and $H$-equivariant maps $f^{m}: X^{m} \rightarrow \bar{X}^{m}$ which are $H$-homotopy equivalences and commute with the inclusions $\iota^{m}: X^{m-1} \rightarrow X^{m}$ and $\imath^{m}: \bar{X}^{m-1} \rightarrow \bar{X}^{m}$. For notational simplicity, we assume that only one $G$-cell is attached at the $n$th stage.

We then have a diagram:

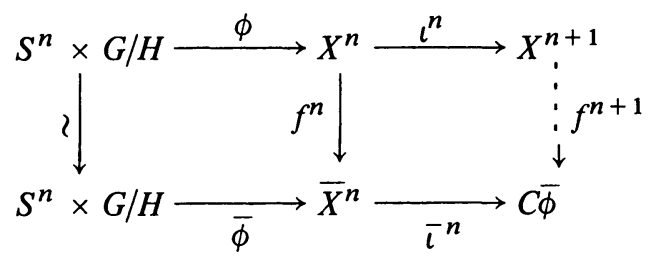

where $\bar{\phi}$ is an $H$-cellular approximation of $f^{n} \circ \phi$, so that the left-hand square $H$-homotopy commutes. With $\bar{X}^{n+1}=C \bar{\phi}$, the existence of $f^{n+1}$ and the fact that it is an $H$-equivalence are purely formal. Now let $\bar{X}=$ colim $\bar{X}^{m}$, which has a natural $H$-CW decomposition. The conclusion then follows by 1.2 .

4. Milnor's Theorem. This section is based largely on [Mi1]. $G$ will be assumed to be compact Lie.

We first introduce a few basic concepts. 
Definition 4.1. A $G$-simplicial complex is a $G$-space $X$ such that $X$ has a given $G$-CW decomposition $X=\operatorname{colim} X^{n}$ such that the orbit space, $X / G$, is a simplicial complex under this structure. All the usual notions about skeleta, stars, links and so on are defined by taking inverse images (under projection) from the orbit space. $G$-simplicial maps are required to have the property that the induced maps on orbit spaces are simplicial in the usual sense, and $G$-subdivisions are defined in the natural way.

Definition 4.2. A $G$-space $X$ is said to be $G$-equilocally convex (GELC) if there is an invariant neighborhood $U$ of the diagonal in $X \times X$ and a $G$-map $\lambda$ : $U \times I \rightarrow X$ which is a homotopy from the first to the second projection through $G$-maps and restricts to a homotopy

$$
\lambda \mid: U_{\alpha} \times U_{\alpha} \times I \rightarrow U_{\alpha}
$$

for some (nonequivariant) cover $\left\{U_{\alpha}\right\}$ of $X$ by open sets such that $W \in\left\{U_{\alpha}\right\}$ implies $W g \in\left\{U_{\alpha}\right\}$ for each $g \in G$. Such a triple $\left(X, \lambda,\left\{U_{\alpha}\right\}\right)$ is called a $G$ ELC representation for $X$, and the sets $U$ are called the convex sets associated with the representation.

The basic examples of $G$ ELC spaces are $G$-orbits $G / H$, for which $G$ ELC representations may be constructed subordinate to any cover $\mathcal{C}$ of $G / H$ which is invariant under the action $W \mapsto W g$ for $g \in G$ and $W \in \mathcal{C}$. (This is done by using linear charts for the manifold $G / H$ and the $G$-action. Alternatively, we may use invariant geodesics to pass from one point to another nearby.) Other important examples are smooth $G$-manifolds supplied with invariant metrics.

$G$ ELC spaces, $G-C W$ complexes and $G$-simplicial complexes are related by the following lemmas:

LEMMA 4.3. Every G-CW complex is G-homotopy equivalent to a colimit, colim $Y_{n}$, of finite dimensional $G$-simplicial complexes taken over cellular inclusions.

A proof appears in $\$ 5$.

LEMMA 4.4. Every finite dimensional G-CW complex is GELC.

A proof appears in $\S 6$.

REMARK 4.5. When $G$ is finite, we may drop the finite dimensionality in 4.4 by adapting the proof of the corresponding result in the nonequivariant case, thus drastically simplifying the proof. The point is that in the finite case, $G$ permutes the cells and the ELC homotopies Milnor defines in [Mi1] are automatically equivariant.

LEMMA 4.6. Let $X$ be a completely regular G-paracompact GELC space. Then $X$ is dominated by a G-CW complex.

A proof appears in $\$ 7$.

Lemma 4.7. Suppose that $X$ is dominated by G-CW complex. Then $X$ has the $G$-homotopy type of a $G-\mathrm{CW}$ complex.

A proof appears in $\$ 5$. 
LEMMA 4.8. Let $X=\operatorname{colim} X_{n}$, taken over a system of $G$-cofibrations such that each $X_{n}$ has the G-homotopy type of a G-CW complex. Then $X$ has the G-homotopy type of a G-CW complex.

The proof is straightforward homotopy theory; replace the colimit by the telescope and use homotopy invariance of the homotopy colimit.

Assuming Lemmas 4.3 to 4.8 , we then have the following:

Theorem 4.9 (Equivariant Version of Milnor's TheOREM). The following are equivalent:

(i) $X$ has the G-homotopy type of a G-CW complex.

(ii) $X$ is G-equivalent to a colimit, colim $X_{n}$, where each $X_{n}$ has the G-homotopy type of a finite dimensional $G$-simplicial complex, and where $X_{n} \rightarrow X_{n+1}$ is a $G$ cofibration for each $n$.

(iii) $X$ is $G$-equivalent to a colimit, colim $X_{n}$ (over G-cofibrations), where each $X_{n}$ has the G-homotopy type of a G-paracompact completely regular GELC space.

(iv) $X$ is G-equivalent to a colimit as above, where each $X_{n}$ is dominated by $a$ G-CW complex.

(v) $X$ is G-equivalent to a colimit as above, where each $X_{n}$ has the G-homotopy type of $a \mathrm{G}-\mathrm{CW}$ complex.

This is an immediate consequence of the above lemmas.

An important application of the above theorem is in the case of function spaces or, more generally, $n$-ads of function spaces. In order to deal with the relative case, we must define relative $G$ ELCs (and $n$-ads of $G$ ELCs).

Definition 4.10. Let $(X, Y)$ be a pair of $G$-spaces. We say that $(X, Y)$ is a relative $G E L C$ if there exists a $G E L C$ representation for $X$ which restricts to one for $Y$. More generally, we may define $G$ ELC $n$-ads of $G$-spaces in a similar manner.

If $\left(X ; X_{1}, \ldots, X_{n}\right)$ and $\left(Y ; Y_{1}, \ldots, Y_{n}\right)$ are $n$-ads of $G$-spaces, we may define the function space $n$-ad of $G$-spaces, $\left(X ; X_{1}, \ldots, X_{n}\right)^{\left(Y ; Y_{1}, \ldots, Y_{n}\right)}$ in the obvious manner, with $G$ acting by conjugation. The following result may be seen from the proof of the corresponding result in [Mi1]:

Proposition 4.11. Let $\left(X ; X_{i}\right)$ be GELC n-ad of G-paracompact completely regular $G$-spaces with each $X_{i}$ closed and invariant, and let $\left(K ; K_{i}\right)$ be an $n$-ad of compact $G$-spaces. Then the function space $n$-ad $\left(X ; X_{i}\right)^{\left(K ; K_{i}\right)}$ is GELC G-paracompact and completely regular. Thus it has the G-homotopy type of a G-CW complex, by Theorem 4.9.

The most important application of 4.11 is the following:

TheOREM 4.12. Let $X$ be an $n$-ad of $G-C W$ complexes, and let $K$ be an $n$-ad of compact $G$-spaces. Then $X^{K}$ has the $G$-homotopy type of an $n$-ad of $G-C W$ complexes.

Proof. Filter the $n$-ad $X^{K}$ by setting $F_{i}\left(X^{K}\right)=\left(X^{i}\right)^{K}$, where $X^{i}$ is the $i$ th skeleton of $X$. Then apply Theorem 4.9 to each of the spaces $F_{i}\left(X^{K}\right)$. 
Other consequences are the following:

Corollary 4.13. Let $X$ and $K$ be as above, and let $\left(X^{K}\right)^{H}$ denote the subspace of $X^{K}$ consisting of $H$-equivariant maps for some closed subgroup $H<G$. Then $\left(X^{K}\right)^{H}$ has the homotopy type of a $\mathrm{CW}$ complex.

Proof. $\left(X^{K}\right)^{H}$ is the fixed-point set of $X^{K}$ by the subgroup $H$, and the result follows by a check that a $G E L C$ representation of a $G$-space restricts to an ELC representation of all its fixed-point sets.

Corollary 4.14. Let $f: X \rightarrow Y$ be a G-map, and let $y \in Y$ have isotropy subgroup $H$. Then, regarding $f$ as an $H$-equivariant map based at $y$, the homotopy-theoretic fiber Ff has the H-homotopy type of an $H$-CW complex whenever $X$ and $Y$ have the $G$-homotopy type of G-CW complexes.

Proof. One observes that $F f=(Y ;\{y\}, X)^{(I ;\{0\},\{1\})}$ if we replace $f$ by an inclusion. Thus the result follows.

5. Proof of Lemmas 4.3 and 4.7.

5.1. Proof of Lemma 4.3. This in turn is proved by lemmas:

LEMMA 5.2. Every G-simplicial complex can be barycentrically G-subdivided subordinately to any given open cover by invariant sets.

Proof. Exactly the same as the $G$-trivial case (e.g., [Sp1]).

LEMMA 5.3. Let $X$ be a $G$-simplicial complex of the form $X=G / H \times Y$, where $Y$ is a nonequivariant simplicial complex, and let $Z$ be an arbitrary $G$-simplicial complex, with $f: X \rightarrow Z$ a G-simplicial map. Then there exists a G-simplicial pair $\left(M^{\prime} f, X\right)$ which is $G$-homotopy equivalent, $\operatorname{rel} X$, to the pair $(M f, X \times\{1\})$, where $M f$ is the usual mapping cylinder of $f$.

Proof. Let $p$ denote projection onto orbit spaces. Then we have a commutative diagram

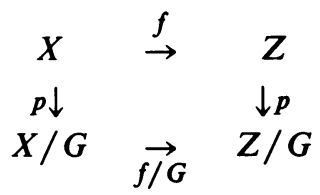

in which $f / G$ is a simplicial map. Let $Z^{\prime}$ be the pullback of $Z$ over $f / G$. Then $Z^{\prime}$ has the structure of a $G$-simplicial complex such that the induced structure on $X / G$ is the given one. Let $\bar{f}: X \rightarrow Z^{\prime}$ be the universal map, and let $g: Z^{\prime} \rightarrow Z$ be the natural map. Then $\bar{f}$ and $g$ are $G$-maps. Observe that $M g$ and $M \bar{f}$ have the structure of $G$-simplicial complexes. Hence $M \bar{f} \cup M g$ is also a $G$-simplicial complex, where $M \bar{f}$ is attached to $M g$ via the inclusion $Z^{\prime} \rightarrow Z^{\prime} \times I \rightarrow M g$. Then $M^{\prime} f=M \bar{f} \cup M g$ is the required space.

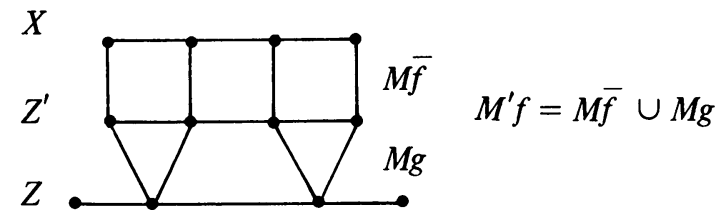


(Note that, when $G$ is not discrete, $M f$ itself need not have a $G$-simplicial structure, even though the orbit space has one: consider, for example, the case $f$ : $S^{1} \times I \rightarrow S^{1}$ given by $f(t, s)=s e^{i t}$, with $G=S^{1}$ and $H=1$.)

LEMMA 5.4. Let $(X, A)$ and $(Y, B)$ be G-simplicial pairs, and let $f: X \rightarrow Y$ be $a$ $G$-map which is simplicial on $A$. Then there is a G-homotope $f^{\prime}$ of $f$ such that $f^{\prime} \mid A=f$, and $f^{\prime} \sim f \operatorname{rel} A$, with $f^{\prime}$ simplicial on $X$ (in some subdivision).

Proof. Using Lemma 5.2 the proof is identical to the nonequivariant case treated in [Sp1].

We may now prove Lemma 4.3: Let $X$ be an arbitrary $G$-CW complex, let $X^{n}$ denote its $n$th skeleton, and assume that we have equivalences

$$
q_{n}: X^{n} \rightarrow \operatorname{colim} Y_{m}^{n} \quad \text { for each } n<N,
$$

where each $Y_{m}^{n}$ is a $G$-simplicial complex of dimension no larger than $n$, where the maps $Y_{m}^{n} \rightarrow Y_{m+1}^{n}$ are given by composites

$$
Y_{m}^{n} \stackrel{B}{\rightarrow} B\left(Y_{m}^{n}\right) \stackrel{i}{\rightarrow} Y_{m+1}^{n},
$$

where $B$ denotes the first barycentric subdivision and where $i$ is inclusion of a subsimplicial $G$-complex. Also assume that each $Y_{m}^{n}$ is a subsimplicial $G$-complex of $Y_{m}^{n+1}$, and that the following diagrams commute up to homotopy for each $n<N$ :

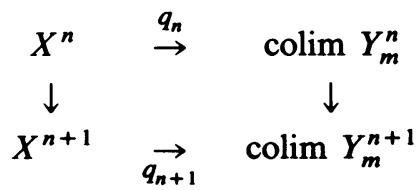

For each $N$-dimensional $G$-cell attached to $X^{N}$, we may use $q_{N}$ to attach it to some $Y_{m}^{N}$. If we subdivide sufficiently many times, we may attach it simplicially to $Y_{m+r}^{N}$ for some $r$, instead, giving the resulting space the structure of a $G$-simplicial complex, by the above lemmas. Further subdivisions also allow us to attach it to $Y_{m+r+s}^{N}$ for each $s$, giving us the complexes $Y_{m}^{N+1}$ for each $m$. Construction of $q_{N+1}$ is now straightforward, completing the inductive construction of the colimits colim $Y_{m}^{n}$ and of the maps $q_{n}$.

Finally, set

$$
Y=\operatorname{colim} Y_{n}^{n} \text {. }
$$

Since the sequence $\left(Y_{n}^{n}\right)$ is cofinal in $\operatorname{colim}_{m}\left(\operatorname{colim}_{n} Y_{m}^{n}\right), Y$ is equivalent to $X$ by invariance of colimits, as required.

5.5. Proof of Lemma 4.7. Suppose that $X$ is dominated by a $G$-CW complex $Y$. Let $\gamma: \Gamma X \rightarrow X$ denote a $G-C W$ approximation of $X$, and let $\mu: X \rightarrow Y$ and $\varepsilon$ : $Y \rightarrow X$ satisfy $\varepsilon \mu \sim 1$. By the Whitehead Theorem, $\varepsilon \sim \gamma \tilde{\varepsilon}$ for some $G$-map $\tilde{\varepsilon}:$ $Y \rightarrow \Gamma X$. Now let $\gamma^{-1}: X \rightarrow \Gamma X$ be the composite $\tilde{\varepsilon} \mu$. One then easily checks that $\gamma \gamma^{-1} \sim 1$, and that $\gamma^{-1} \gamma$ is a weak-, and hence strong, $G$-homotopy equivalence. From this it follows that $\gamma$ is a $G$-homotopy equivalence as required. 
6. Finite dimensional $G$-simplicial complexes are $G$ ELC. In this section, Lemma 4.4 is proved. First, we shall need some information about "barycentric convexity".

Definition 6.1. A subset $C$ of a simplicial complex $K$ is barycentrically convex if, whenever $k_{1}$ and $k_{2}$ are in $C$, then so is $t k_{i}+(1-t) \mu\left(k_{1}, k_{2}\right)$ for $t \in I$ and $i=1,2$, where $\mu\left(k_{1}, k_{2}\right)$ is the point whose $\gamma$ th barycentric coordinate is

$$
\frac{\min \left\{k_{1 \gamma}, k_{2 \gamma}\right\}}{\sum_{\gamma \in \Gamma} \min \left\{k_{1 \gamma}, k_{2 \gamma}\right\}} .
$$

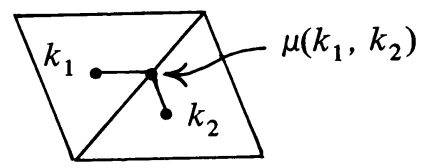

The average of $k_{1}$ and $k_{2}$.

( $\Gamma$ is the set of vertices in $K$, and $k_{i}$ is the $\gamma$ th barycentric coordinate of $k_{i}$ )

(It follows therefore that $k_{1}$ and $k_{2}$ must both lie in some star $S_{\gamma}$ of a vertex $\gamma$.) Obvious examples of barycentrically convex sets are open and closed stars. A slightly less obvious example is the following.

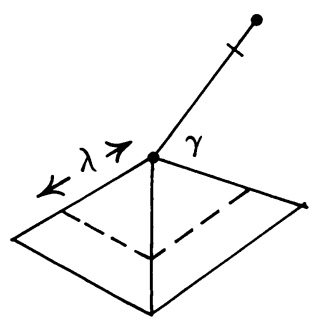

The $\lambda$-star of $\gamma$.

Let $\lambda \in(0,1)$. The $\lambda$-star of a vertex $\gamma_{0}$ in $K$ is the set of points in the star of $\gamma_{0}$ whose $\gamma_{0}$ th coordinates are greater than $\lambda$. $\lambda$-stars are barycentrically convex simply because $\Sigma_{\gamma \in \Gamma} \min \left\{k_{1 \gamma}, k_{2 \gamma}\right\} \leqslant 1$ for any pair $\left(k_{1}, k_{2}\right)$ of points in $K$. (Note that $\Sigma_{\gamma \in \Gamma} k_{\gamma}=1$ for $k=\left(k_{\gamma}\right)$.) Intersections of $\lambda$-stars of the first barycentric subdivision will be used in constructing convex sets for $G$-simplicial complexes.

We are now ready to prove 4.4. As remarked earlier, when $G$ is finite, one simply observes that the first barycentric subdivision of any $G$-simplicial complex is a $C W$ complex on which $G$ acts by permuting the cells. (In fact, it is a regular $G$-complex as described in Bredon [Br1].) It is then easy to verify that the ELC representation described in [Mi1], applied to any subdivided $G$-simplicial complex, yields a $G$ ELC representation. As a consequence, we may ignore all countability assumptions made here when dealing with finite group actions. When $G$ is not finite, we have to take local convexity of the orbits into account, and it does not seem at all likely 
that we can choose a system of $G$ ELC representations $\left\{\left(G / H, \lambda_{H},\left\{U_{H_{\alpha}}\right\}\right): H<\right.$ $G$ ) compatibly (in the sense that for every $G$-map $\phi: G / H \rightarrow G / K$ there are commutative diagrams):

$$
\begin{array}{ccc}
U_{H_{\alpha}} \times U_{H_{\alpha}} \times I & \stackrel{\lambda_{H}}{\rightarrow} & U_{H_{\alpha}} \\
\downarrow \phi \times \phi \times 1 & & \downarrow_{\phi} \\
U_{K_{\beta}} \times U_{K_{\beta}} \times I & \overrightarrow{\lambda_{K}} & U_{K_{\beta}}
\end{array}
$$

Thus the "obvious" generalization of the $G$ finite case cannot possibly work. We must therefore be able to pass continuously from one GELC representation $\left(G / H, \lambda_{H},\left\{U_{H_{\alpha}}\right\}\right)$ to another, possibly incompatible, representation $\left(X^{n-1}, \lambda,\left\{U_{\alpha}\right\}\right)$ whenever an $n$-simplex $\Delta^{n} \times G / H$ is attached to a given $G$-simplicial complex $X^{n-1}$. This is achieved by using a deformation $\theta$ of the outer rim of $\Delta^{n}$ to its boundary, and then using $\lambda$ to pass from the image of $\lambda_{H}$ (under this deformation) to $\lambda$ itself. Use of $\theta$ precludes taking the convex sets to include the barycentric coordinates of a whole star as our convex sets, so we shall have to take smaller sets to obtain $\theta$-invariance, and make sure that they are also invariant under the various homotopies defining the convexity property.

We proceed now to the proof.

Proof of Lemma 4.4. If $X$ is a disjoint union of $G$-spaces of the form $G / H_{\gamma}$, for varying $\gamma$, then $X$ is certainly $G$ ELC by the remarks after 4.2 .

Proceeding inductively on dimension, we may assume that $X$ is obtained from an $n$ - 1 dimensional complex $Y$ by attaching $G$-simplices, and that we have a $G$ ELC representation $\left(Y, \lambda,\left\{U_{\alpha}\right\}\right)$ for $Y$. Let $\phi: \partial \Delta^{n} \times G / H \rightarrow Y$ denote a typical attaching map.

Consider the open cover $\left\{\phi^{-1}\left(U_{\alpha}\right)\right\}$ of $\partial \Delta^{n} \times G / H$. Since $G / H$ is $G$ ELC, we may choose a representation $\left(G / H, \chi,\left\{V_{\beta}\right\}\right)$ and an $\varepsilon>0$ such that if $B$ is any $\varepsilon$-ball in $\partial \Delta^{n}$, then $\phi\left(B \times V_{\beta}\right)$ lands in some $U_{\alpha}$. Assume $\varepsilon<\frac{1}{2}$. Choose a finite cover of $\Delta^{n}$ by open (in $\Delta^{n}$ ) simplices of diameter $<\varepsilon$ whose faces are parallel with those of $\Delta^{n}$ itself, and then let $\left\{\tilde{S}_{\gamma}\right\}$ denote the set of those simplices that meet $\partial \Delta^{n}$. We may then replace $\left\{\tilde{S}_{\gamma}\right\}$ by a subordinate finite collection of such simplices, $\left\{S_{\gamma}\right\}$ such that $\left\{\dot{S}_{\gamma} \cap \partial \Delta^{n}\right\}$ is still a cover of $\partial \Delta^{n}$, and such that $S_{\gamma} \cap S_{\gamma^{\prime}}$ is a simplex which meets $\partial \Delta^{n}$ in a single face, or is empty, for each $\gamma$ and $\gamma^{\prime}$. It follows that for each $\gamma, \phi\left(\left(\dot{S}_{\gamma} \cap \partial \Delta^{n}\right) \times V_{\beta}\right)$ lands in some $U_{\alpha}$ for every $\gamma$ and $\beta$.

Since $\left\{S_{\gamma}\right\}$ is finite, we can find numbers $0<r_{1}<r_{2}<r_{3}<\operatorname{diam}\left(\Delta^{n}\right)$ such that if $\Delta_{i}^{n} \subset \Delta^{n}$ is the simplex concentric and parallel with $\Delta_{n}^{i}$ of diameter $r_{i}$, then $\left\{\dot{S}_{\gamma} \cap \Delta_{i}^{n}\right\}$ forms a cover of $\partial \Delta_{i}^{n}(i=1,2,3)$.

Since the $S_{\gamma}$ intersect appropriately, we may choose a homotopy $\theta_{t}: \Delta^{n} \rightarrow \Delta^{n}$ which deforms $\Delta_{2}^{n}$ to the boundary keeping $\partial \Delta^{n}$ and $\Delta_{1}^{n}$ fixed such that each $\dot{S}_{\gamma}$ is $\theta_{t}$ invariant, and such that the curves $\theta_{t}(x)$ approach the boundary montonically and with $\theta_{t}(x)$ closer to $\partial \Delta^{n}$ than, and on the same curve $\theta_{s}(y)$ as, $y$ wherever $\theta_{t_{0}}(y)=x$ for some $t_{0} \in I$ (see Figure 1).

Now define open sets of $Y$ as follows: 


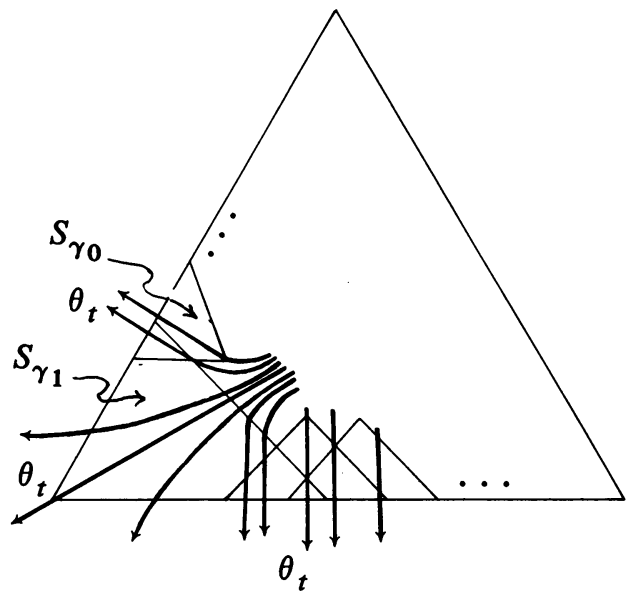

Figure 1. The Simplices $S_{\gamma}$ and the Homotopy $\theta_{t}$

For each triple $\mu=(\gamma, \alpha, \beta)$ such that $\phi\left(\left(\dot{S}_{\gamma} \cap \partial \Delta^{n}\right) \times V_{\beta}\right)$ lands in $U_{\alpha}$, set

$$
R_{\mu}=\left(\left(\phi \circ \theta_{1}\right)^{-1}\left(U_{\alpha}\right) \cap\left(\left(\Delta^{n}-\delta_{2}^{n}\right) \times G / H\right)\right) \cup \theta_{1}^{-1}\left(S_{\gamma} \times V_{\beta}\right) \text {. }
$$

(See Figure 2.)

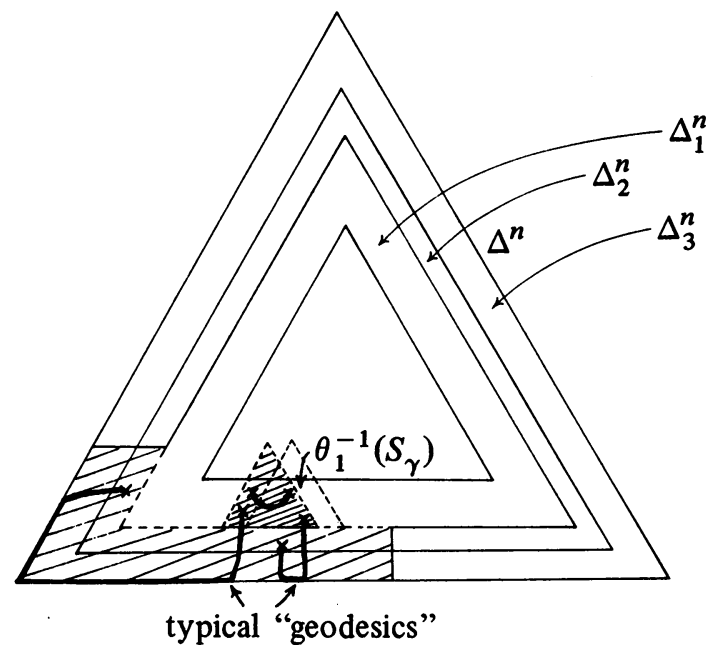

FIgURE 2. The Sets $p\left(R_{\mu}\right) \subset X / G$

Now $R_{\mu}$ is $\theta_{t}$-invariant for all $t$, because of the properties of $\theta_{t}$.

For each $U_{\alpha}$, also let

Finally, let $T=\AA_{1}^{n}$.

$$
W_{\alpha}=\left(\phi \circ \theta_{1}^{-1}\right)\left(U_{\alpha}\right) \cap\left(\Delta^{n}-\Delta_{2}^{n}\right) \times G / H .
$$

Our convex sets $U_{\alpha}^{n}$ will consist of the sets $T$, the sets $W_{\alpha}$ (for all the $n$-dimensional $G$-simplices being attached) and unions $R_{\mu_{1}} \cup R_{\mu_{2}} \cup \cdots$ with each $R_{\mu_{1}}$ in a 
distinct $n$-dimensional $G$-simplex, $G / H_{i} \times \Delta^{n}$ such that they intersect $Y$ in the same convex set $U_{\alpha}$, and such that the resulting sets are open in $X$. The collection $\left\{U_{\alpha}^{n}\right\}$ then forms a $\theta_{t}$-invariant open cover of $X$.

Since there are only finitely many little simplices $S_{\gamma}$ in each $n$-simplex, we can find a deformaton $\tilde{\theta}_{t}: \Delta^{n} \rightarrow \Delta^{n}$ for each $n$-simplex which deforms each $S_{\gamma}$ into the boundary such that each $\stackrel{\circ}{\gamma}_{\gamma}$ is $\tilde{\theta}_{t}$-invariant for all $t$.

We shall define the required GELC homotopies $\lambda_{\alpha}^{n}: U_{\alpha}^{n} \times U_{\alpha}^{n} \times I \rightarrow U_{\alpha}^{n}$ by a succession of five homotopies $\mu_{i}$ which have the following effect:

(i) $\mu_{1}$ homotopes the first projection to the first projection followed by $\theta_{1}$. Schematically, we represent this by $p \leadsto \theta_{1}(p)$ (for a pair $(p, q) \in U_{\alpha}^{n} \times U_{\alpha}^{n}$ ).

(ii) $\mu_{2}$ applies $\tilde{\theta} ; \tilde{\theta}_{1}$ to everything outside $\Delta_{2}^{n}$, and $\tilde{\theta}_{0}=1$ to everything inside $\Delta_{1}^{n}$. We thus obtain $\theta_{1}(p) \leadsto \tilde{\theta}_{r t} \theta_{1}(p)$ for some $r \in I$.

(iii) In this stage, we pass continuously from a $G$ ELC representation for the new cell to the one given inductively by using $\lambda_{t} \circ \tilde{\theta}_{1} \circ \theta_{1}$. The new GELC representation is given by first averaging the barycentric coordinates (as in [Mi1]) keeping the orbit coordinate fixed (up to the action of the attaching map), then using $\left(G / H, \chi,\left\{V_{\beta}\right\}\right)$ to get around the image of $G / H$, and finally going to $\tilde{\theta}_{r t} \theta_{1}(q)$. Schematically, this is

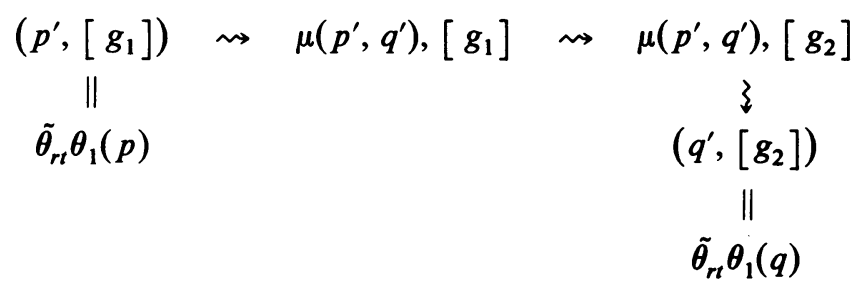

Stages (iv) and (v) are, respectively, the mirror images of (ii) and (i).

Proceeding formally, we define the $\mu_{i}$ as follows:

(i) $\left(\mu_{1}\right)_{t}=\theta_{t} \circ \pi_{1}$ where $\pi_{1}$ is the first projection.

(ii) $\left(\mu_{2}\right)_{t}\left(x,[q], y,\left[g^{\prime}\right]^{\prime}\right)=\tilde{\theta}_{r t}\left(\theta_{1}(x),[q]\right)$, where

$$
r=\min \left\{\max \left\{d\left(\mu\left(\theta_{1}(x), \theta_{1}(y)\right)\right), 0\right\}, 1\right\},
$$

$\mu(p, q)$ is the average of $p$ and $q$, and $1-d(p)$ is the ratio

$$
\text { distance }\left(p, \Delta^{n}-\Delta_{2}^{n}\right) / \text { distance }\left(\Delta_{1}^{n}, \partial \Delta_{2}^{n}\right)
$$

(for some metric on $X$ ).

(iii) $\left(\mu_{3}\right)_{t}\left(x,[g], y,\left[g^{\prime}\right]^{\prime}\right)=\rho_{t}\left(\tilde{\theta}_{1} \theta_{1}(x),[g], \tilde{\theta}_{1} \theta_{1}(y),\left[g^{\prime}\right]^{\prime}\right)$ if $\mu\left(\theta_{1}(x), \theta_{1}(y)\right) \in$ $\Delta_{2}^{n}, \lambda_{s}\left(\rho_{t}\left(\tilde{\theta}_{1} \theta_{1}(x),[g], \tilde{\theta}_{1} \theta_{1}(y),\left[g^{\prime}\right]^{\prime}\right)\right), \lambda_{t}^{n}\left(\tilde{\theta}_{1} \theta_{1}(x),[g], \tilde{\theta}_{1} \theta_{1}(y),\left[g^{\prime}\right]^{\prime}\right)$ if $\mu\left(\theta_{1}(x), \theta_{1}(y)\right)$ $\in \Delta_{3}^{n}-\check{\Delta}_{2}^{n}$ (where $s$ is the ratio)

$$
\begin{aligned}
& \text { distance }\left(\mu(x, y), \Delta_{2}^{n}\right) / \text { distance }\left(\Delta_{2}^{n}, \partial \Delta_{3}^{n}\right) \text {; } \\
& \lambda_{t}\left(\tilde{\theta}_{1} \theta_{1}(x),[g], \tilde{\theta}_{1} \theta_{1}(y),\left[g^{\prime}\right]^{\prime}\right) \text { if } \mu\left(\theta_{1}(x), \theta_{1}(y)\right) \in \Delta^{n}-\dot{\Delta}_{3}^{n} \text {. } \\
& \text { Here, } \rho_{t}=\rho_{t}^{1}+\rho_{t}^{2}+\rho_{t}^{3} \text { where } \\
& \rho_{t}^{1}\left(x,[g], y,\left[g^{\prime}\right]^{\prime}\right)=\left((1-t) \tilde{\theta}_{1} \theta_{1}(x)+t \mu(x, y),[g]\right) ; \\
& \rho_{t}^{2}\left(x,[g], y,\left[g^{\prime}\right]^{\prime}\right)=\left(\mu(x, y), \chi_{t}\left([g],\left[g^{\prime}\right]^{\prime}\right)\right) ; \quad \text { and } \\
& \rho_{t}^{3}\left(x,[g], y,\left[g^{\prime}\right]^{\prime}\right)=\left(t \tilde{\theta}_{1} \theta_{1}(y)+(1-t) \mu(x, y),\left[g^{\prime}\right]^{\prime}\right) \text {. }
\end{aligned}
$$


(We are also identifying the points $(x,[g])$ and $\left(y,\left[g^{\prime}\right]^{\prime}\right)$ with their images in $y$.)

(iv) is (ii) reversed, with $y$ and $\left[g^{\prime}\right]^{\prime}$ replacing $x$ and $[g]$.

(v) is $\theta_{1-t} \circ \pi_{2}$.

It is then straightforward to check that $\left(Y, \lambda^{n},\left\{U_{\alpha}^{n}\right\}\right)$ is the required GELC representation. This completes the inductive step, and the proof.

7. Reasonable $G$ ELC spaces are dominated by $G-C W$ complexes. In this section, 4.6 is proved. The following types of spaces will be used for the construction of $G$-CW complexes which dominate suitable $G$-spaces.

Definition 7.1. Let $\theta=\left\{Q_{\alpha}\right\}_{\alpha \in A}$ be a set of $G$-orbits (of not necessarily distinct type). The indexed reduced join, $J(\theta)$ of $\theta$ is defined to be the geometric realization of the simplicial space $J_{*}(\theta)$ whose $n$th space is given by

$$
J_{n}(\vartheta)=\left\{\left(x_{0}, \ldots, x_{n}\right) \in\left(\underset{\alpha}{\amalg} Q_{\alpha}\right)^{n+1}: i \neq j \Rightarrow x_{i}\right.
$$

$$
\text { and } \left.x_{j} \text { lie in distinct summands }\right\} \text {, }
$$

and the $i$ th face operator is given by omitting the $i$ th term. There is no degeneracy, and $G$ acts diagonally on $J_{n}(\theta)$.

Proposition 7.2. Let $\theta$ be a set of G-orbits, and let $J_{*}^{\prime}(\theta)$ be a subsimplicial $G$ space of $J_{*}(\theta)$. Then $J^{\prime}(\theta)$ is in $G$ W whenever each $J_{n}^{\prime}(\theta)$ is in $G$ W.

Proof. Let $F$ denote the standard filtration of $J^{\prime}(\theta)$. Any representation of $\left(\Delta^{n}, \partial \Delta^{n}\right)$ as an N.D.R. pair gives rise to a $G$-N.D.R. representation for $F_{n}\left(J^{\prime}(\theta)\right)$, $F_{n-1}\left(J^{\prime}(\theta)\right)$. Thus the maps $i_{n-1}: F_{n-1}\left(J^{\prime}(\theta)\right) \rightarrow F_{n}\left(J^{\prime}(\theta)\right)$ are $G$-cofibrations.

By 1.2 , it suffices to construct a homotopy commutative diagram:

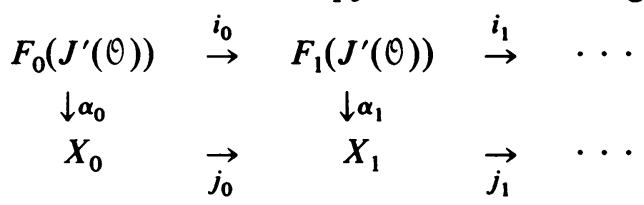

in which each $\alpha_{r}$ is a $G$-homotopy equivalence, each $X_{i}$ is a $G-\mathrm{CW}$ complex, and each $j_{r}$ is a $G$-cellular inclusion.

Inductively, let $X_{0}=F_{o}\left(J^{\prime}(\theta)\right)$, let $\alpha_{0}$ be the identity, and suppose that $X_{n-1}$, $\alpha_{n-1}$ and $j_{n-1}$ have been defined with the required properties. Consider the following diagram, in which both inner squares are pushouts:

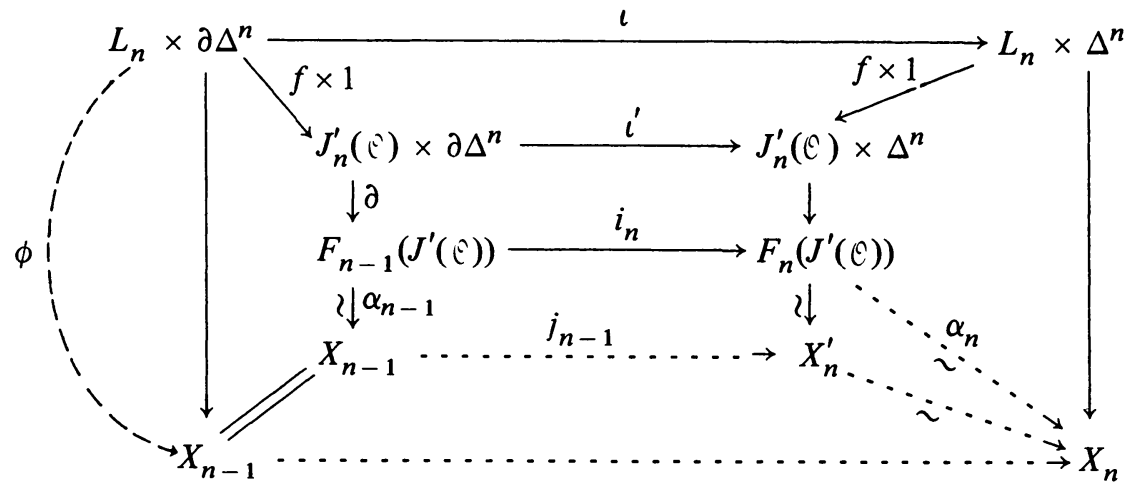


Here, $L_{n}$ is a $G$-CW approximation to $J_{n}^{\prime}(\theta), X_{n}^{\prime}$ is the pushout of $i_{n}$ and $\alpha_{n-1}$ and $\phi$ is a $G$-cellular approximation of $\alpha_{n-1} \circ \partial \circ(f \times 1)$. This data gives us the dotted arrows making the outer square and the trapezoid on the right $G$-homotopy commutative, $X_{n}$ being the double mapping cylinder of $\iota$ and $\phi$, which has the structure of a $G-\mathrm{CW}$ complex.

Definition 7.3. If $\theta: V \times{ }_{H} G \rightarrow X$ is an embedding of a tube, and if $S \subset$ $G / H$, then we call $\theta \mid p^{-1}(\delta)$ the tube segment generated by $S$. Here $p: V \times{ }_{H} G \rightarrow$ $G / H$ is the projection.

When $X$ is a completely regular $G$-space, the open tube segments form a base for the topology of $X$ (by the topological slice theorem [Br1] and compactness of $G$ ).

We now prove Proposition 4.6.

Proof of Proposition 4.6. In view of 7.2, it suffices to show that $X$ is dominated by a $G$-space of the form $J^{\prime}(\theta)$ as described there.

Let $\left\{U_{\alpha}\right\}_{\alpha \in A}$ be a cover of $X$ by convex sets forming part of a GELC representation $\left(X, \lambda,\left\{U_{\alpha}\right\}\right)$, and let $\left\{V_{\beta}\right\}_{\beta \in B}$ be a subordinate cover by open tube segments. Then $\left\{V_{\beta} G\right\}$ is an open cover of $X$ by invariant open sets. By $G$-paracompactness, there exists a locally finite cover of $X$ by open tubes, $\left\{T_{\gamma}\right\}_{\gamma \in \Gamma}$, which is so fine that the star of any point is contained in some $V_{\beta} G$, and hence in some $U_{\alpha} G$.

For each $\gamma \in \Gamma$, let $Q_{\gamma}$ denote the central orbit of $T_{\gamma}$. Then there are open subspaces $P_{\gamma}$ of $Q_{\gamma}$ such that if $R_{\gamma}$ denotes the segment generated by $P_{\gamma}$, then the star of every point in $X$, taken among the sets $R_{\mu} g$ with $\mu \in \Gamma$ and $g \in G$, is contained in some $V_{\gamma}$. Recalling that $U_{\alpha} g \in\left\{U_{\alpha}\right\}$ for every $g \in G$, we define $J_{n}^{\prime}(\theta) \subset\left(\amalg_{\gamma \in \Gamma} Q_{\gamma}\right)^{n+1}$ as follows:

For each $n$, let $J_{n}^{\prime}(\theta)$ be the union over all $\gamma_{i}$ of the sets $\left(P_{\gamma_{0}} g_{0} \times \cdots \times P_{\gamma_{n}} g_{n}\right) G$ with $\left(R_{\gamma_{0}} g_{0} \cup \cdots \cup R_{\gamma_{n}} g_{n}\right)$ included in the star of some point in $X$.

Then $J_{n}^{\prime}(\Theta)$ satisfies the hypothesis of 7.2. ${ }^{1}$

We may assume that the $T_{\gamma}$ are enumerated by a $G$-partition of unity, so that there is a natural map $\theta: X \rightarrow J^{\prime}(\theta)$ defined by this partition. It thus suffices to show that $\theta$ is a homotopy section of some $G$-map $q: J^{\prime}(\theta) \rightarrow X . q$ will be defined inductively.

Order the set and give $J^{\prime}(\theta)$ a filtration $F$, by defining $F_{n}\left(J^{\prime}(\theta)\right) \subset J^{\prime}(\theta)$ to be the $n$th simplicial filtration. Since the inclusions $F_{n}\left(J^{\prime}(\theta)\right) \rightarrow F_{n+1}\left(J^{\prime}(\theta)\right)$ are $G$-cofibrations, we have $J^{\prime}(\theta)=$ colim $F_{n}\left(J^{\prime}(\theta)\right)$.

There is an obvious map $q_{0}: F_{0} J^{\prime}(\theta) \rightarrow X$. Suppose, then, that $q_{n-1}: F_{n-1} J^{\prime}(\theta)$ $\rightarrow X$ has been defined and extends $q_{n-2}$. Let $\left(\left([g]_{\gamma_{0}}, t\right),(1-t) x\right)$ be a point in $F_{n} J^{\prime}(\theta)$, where $\gamma_{0}$ is smaller than each index appearing in $x$. Then set

$$
q_{n}\left(\left([g]_{\gamma_{0}}, t\right),(1-t) x\right)=\lambda\left([g]_{\gamma_{0}}, q_{n-1}(x), t\right) .
$$

The fact that $[g]_{\gamma_{0}}$ and $q_{n-1}(x)$ are in the same convex set follows from the definition of $J^{\prime}(\theta)$ and the induction hypothesis. This gives $q=$ colim $q_{n}$.

\footnotetext{
${ }^{1}$ See the note at the end of the proof.
} 
Finally, let $H: 1 \sim q \theta$ be given by $(x, t) \mapsto \lambda(x, q \theta(x), t)$. In order to see why $q \theta(x)$ and $x$ both lie in some convex set, we note that the sets $\left(R_{\gamma_{0}} g_{0} \cup R_{\gamma_{1}} g_{1}\right.$ $\left.\cup \cdots \cup R_{\gamma_{m}} g_{m}\right)$ form a cover of $X$, and that if $x \in\left(R_{\gamma_{0}} g_{0} \cup R_{\gamma_{1}} g_{1}\right.$ $\left.\cup \cdots \cup R_{\gamma_{m}} g_{m}\right)$, then $q \theta(x)$ lies in any $U_{\alpha}$ for which $\left(R_{\gamma_{0}} g_{0} \cup R_{\gamma_{1}} g_{1}\right.$ $\left.\cup \cdots \cup R_{\gamma_{m}} g_{m}\right) \subset U_{\alpha}$.

This completes the proof.

REMARK 7.4. Although it is folklore that smooth $G$-manifolds have the structure of $G-\mathrm{CW}$ complexes, there appears to be no rigorous proof in the literature for $G$ compact Lie.

The spaces $J_{n}^{\prime}(\theta)$ in the above proposition are smooth $G$-manifolds, and it would suffice to show that they have the $G$-homotopy type of $G-C W$ complexes. This may be seen by exploiting the existence of $G$-handlebody decompositions of smooth $G$-manifolds [Ws1] and arguing by induction over dimension, as suggested by Rothenberg (private communication), the key point being that the unit disc of a representation is the cone of a smooth $G$-manifold of lower dimension.

On the other hand, we may avoid handle decompositions entirely by arguing as follows: There are natural projections from the components of $J_{n}^{\prime}(\theta)$ onto any one of the orbits $G / H_{\gamma}$ occurring in that component. Thus $J_{n}^{\prime}(\theta)=G \times{ }_{H} Y$ for some closed subgroup $H<G$. For nontriviality, assume that $H \neq G$. We then assume, by induction over closed subgroups of $G$ that Milnor's Theorem holds for the proper subgroup $H<G$. Then $Y$, being a smooth $H$-manifold, is automatically $H$ ELC, and hence has the $H$-homotopy type of an $H$-CW complex. But then $G \times{ }_{H} Y$ then has the $G$-homotopy type of a $G-C W$ complex, since any $H$-CW decomposition of an $H$-homotope $Y^{\prime}$ of $Y$ determines a natural $G$-CW structure for $G \times{ }_{H} Y^{\prime}$. Thus we may carry on to establish Proposition 4.6, and hence Milnor's Theorem.

\section{REFERENCES}

[Br1] G. E. Bredon, Introduction to compact transformation groups, Academic Press, New York, 1972.

[II1] S. Illman, Equivariant singular homology and cohomology, Mem. Amer. Math. Soc., no. 156 (1975).

[Mi1] J. Milnor, On spaces having the homotopy type of a CW complex, Trans. Amer. Math. Soc. 90 (1959), 272-280.

[Mo1] T. Matumoto, Equivariant $K$-theory and Fredholm operators, J. Fac. Sci. Tokyo 18 (1971/72), $109-112$.

[Mo2] , On G-CW complexes and a theorem of J. H. C. Whitehead, J. Fac. Sci. Tokyo 18 (1971/72), 363-374.

[Sp1] E. H. Spanier, Algebraic topology, McGraw-Hill, New York, 1966.

[Ma1] J. P. May, Homotopic foundations of algebraic topology, Mimeographed notes, Univ. of Chicago (to appear).

[Wa1] S. Waner, Equivariant classifying spaces and fibrations, Thesis, Univ. of Chicago, 1978.

[Wa2] _ Classification of oriented spherical fibrations (in preparation).

[Ws1] A. G. Wasserman, Equivariant differential topology, Topology 8 (1969), 127-150.

[LW] C. Lee and A. G. Wasserman, On the groups JO(G), Mem. Amer. Math. Soc., no. 159 (1975).

Department of Mathematics, Princeton University, Princeton, New Jersey 08540 